\title{
La producción escrita del Departamento de Psicología en la Universidad de Puerto Rico entre 1924-1943: Estudio preliminar ${ }^{1}$
}

\section{Written works in the Psychology Department of the University of Puerto Rico between 1924-1943: Preliminary Study}

\author{
Sofía González-Rivera* \\ Universidad del Sagrado Corazón, San Juan, Puerto Rico.
}

\begin{abstract}
RESUMEN
Este trabajo esboza los hallazgos iniciales de una investigación dirigida a identificar y contextualizar la psicología que practicaron aquellos que laboraron a principios del siglo XX en el primer Departamento de Psicología en Puerto Rico. A través de una revisión histórica de documentación oficial universitaria y producción escrita de quienes laboraron en dicho departamento se especifican características de la disciplina entre 1924-1943. El contexto socio-político es tomado en consideración en el momento de interpretar los resultados recopilados. Varias tendencias pueden identificarse a partir de los fuentes analizadas: un fuerte desarrollo en el área de psicometría educativa, preferencia por el sistema funcionalista estadounidense, un vínculo con la Universidad de Columbia y la concordancia entre los intereses del Comisionado de Educación y la producción empírica del profesorado.

Palabras Claves: Departamento de Psicología, Historia, Puerto Rico.
\end{abstract}

\begin{abstract}
Through the analysis of the writing of those who worked in the first Psychology Department at Puerto Rico, we try to identify the characteristics of this discipline in the island between the years of 1924 through 1943. In order to interpret our first finding, this investigation takes into account the social-political context of the country. Several trends can be identified from the data collected: a strong development in the area of educational psychometrics, preference for the American functionalism system, a link with the Columbia University and the harmony between the interests of the Commissioner of Education and the empirical production of the faculty.

Keywords: Psychology Department, History, Puerto Rico.
\end{abstract}

\section{Introducción}

Este trabajo es solo un esbozo de los primeros hallazgos de una investigación que aspira a demarcar y contextualizar la disciplina que practicaron aquellos que laboraron en el primer departamento de psicología en la Universidad de Puerto Rico. Para comenzar nuestra 
investigación histórica examinamos diversos fondos documentales que se conservan en el Archivo Central-Histórico de la institución. Analizamos los informes anuales disponibles del departamento de psicología (1932-1943), de la facultad de pedagogía (1931-1943), la escuela elemental (1931-1941) y la escuela superior (1937-1940) de la universidad. Evaluamos los expedientes académicos de aquellos que impartieron clases e investigaron en la dependencia en cuestión. Asimismo, empezamos a recopilar los catálogos anuales y anuncios de ofertas académicas que están en la Oficina del Registrador. Para contextualizar los hallazgos empleamos periódicos, revistas y libros del periodo en la medida que proporcionaban detalles que nos facilitaban ir adentrándonos en el espíritu de la época. Esta fase de documentación inicial también incluyó libros y artículos sobre historia de Puerto Rico, historia de la Universidad e historia de la psicología en Puerto Rico, entre otras fuentes secundarias. Las referidas fuentes primarias nos han permitido ir compilado un legado que se hallaba disperso; las conferencias, los libros, los artículos en prensa y revistas de Fred Walters, Teobaldo Casanova, Malvina Monefeld, Ramón Ramírez López, Ismael Rodríguez Bou, Mercedes Chiqués Walsh, Néstor Vincenty, Alfredo Silva, Julio Sellés Solá, Eva Milán Martínez y Efraín Sánchez Hidalgo. Este grupo de personas, en su mayoría psicólogos educativos, formó la facultad de este departamento inicial cuya historia está entrelazada a las transformaciones en la educación que se emprendieron en Puerto Rico bajo el régimen norteamericano.

\section{Práctica y contexto: anotaciones}

La invasión norteamericana en 1898 supuso una reestructuración del estado educativo puertorriqueño. Convencidos de que la instrucción escolar era un instrumento útil para americanizar, los recién llegados asignaron más recursos para sufragar la educación que la antigua administración española. El tema de la educación cobró importancia en un sistema que se movía de un viejo orden feudal de haciendas hacia un sistema capitalista agrario (Scarano, 2002). Año tras año, aumentaron la cantidad de planteles, estudiantes y maestros en el sistema de instrucción pública, especialmente, en los niveles elementales. Con el propósito de satisfacer la demanda de maestros en la Isla, el gobierno militar estadounidense ubicó en 1900 una Escuela Normal en el este del país. Un año después la Escuela Normal, -Colegio de Educación de la Universidad de Puerto Rico después de 1923-, fue trasladada a Río Piedras. En sus primeros diez años "la Normal" graduó el 95\% de los 2,665 egresados de la Universidad de Puerto Rico. Aunque los nuevos colegios y escuelas, Farmacia (1913), Leyes (1913), Artes Liberales (1910), 
Administración Comercial (1926) y Escuela de Medicina Tropical (1926), - fueron restando a este porcentaje, un número considerable del estudiantado formó parte del Colegio de Educación en los años bajo estudio (Escuela Normal Insular, 1902; Rodríguez, 1991; Scarano, 2002). El Colegio de Educación fue importante en este periodo, no sólo porque posibilitaba la formación de los profesionales que tenían como labor facilitar el proceso de adaptación de la ciudadanía a los cambios socio-económicos que se estaban gestando para estos años en la Isla, sino también porque el magisterio comenzó a representar una oportunidad de ascenso en los estratos sociales, primordialmente para las mujeres (Batista, 1967; Massó, 1932).

Situado en el Colegio de Educación, el departamento de psicología tuvo desde sus comienzos en la década del veinte una amplia matrícula. Empero, la mayoría de las secciones estaban limitadas a los cursos requisitos para los estudiantes que ejercerían el magisterio: General Psychology, Educational Psychology y Tests y Measurements. No es hasta finales de la década del treinta que se amplían los ofrecimientos y aparece en el catálogo de la universidad la posibilidad de una concentración en la disciplina. En cuanto a los temas que se tocaban en los cursos antes mencionados estaban memoria, instintos, hábitos, atención, psicología anormal, percepción, aprendizaje, diferencias individuales, motivación, emoción, medición y experimentación (Department of Psychology, 1940; Universidad de Puerto Rico, 1915; 1917; University of Puerto Rico, 1924).

Hay que señalar, sin embargo, que la enseñanza de la psicología en la isla no es posterior a la llegada estadounidense. Bajo el dominio español se impartieron cursos sobre psicología, lógica y filosofía moral en el Instituto Civil de Segunda Enseñanza de Puerto Rico (Instituto Civil de Segunda Enseñanza de Puerto Rico, 1874). Así también, desde 1900, la psicología formó parte del currículo de la ya mencionada Escuela Normal (González, 1982). En las primeras décadas del pasado siglo en el Colegio de Artes Liberales y en la Escuela de Leyes, igualmente se dictaron cursos de psicología en la Universidad. No obstante, fue en el Colegio de Educación donde hasta 1943 hubo la oferta curricular mayor para aquellos estudiantes interesados en la materia (Álvarez, 2006b).

El contenido de la oferta curricular apunta hacia el funcionalismo como el sistema psicológico de mayor importancia en Puerto Rico durante las primeras décadas del siglo XX. La inclinación hacia el primer sistema de la adaptación estadounidense será evidente en la descripción de los cursos de Psicología General, Psicología General Avanzada y Psicología Pedagógica los que hacen referencia al funcionalismo como la estructura teórica en donde se enmarcaría la discusión de la clase. De igual manera, la vigencia del funcionalismo, 
será visible en la elección de los textos no sólo para los cursos de psicología, sino también para muchos otros cursos del Colegio de Educación. En General Psychology, Methods and Pedagogy y Principles of Education se asignaban Elements of psychology, How to teach fundamental subject y Education respectivamente; todos de Thorndike. Ethics de Dewey and Tufts era el texto para el curso de Ética. En Introductory Psychology que se ofrecía en 1925 para estudiantes de Leyes se utilizaba Instruction of psychology de Angell. $^{2}$ Apartándose un tanto del resto del currículo, en Liberal Art Psychology se estudiaban los temas propios del psicoanálisis: la histeria, el hipnotismo y los sueños. Naturalmente, evidencia robusta adicional es necesaria antes de poder establecer como hipótesis que el psicoanálisis o cualquier otra escuela constituyó una fuerza importante en Puerto Rico durante las primeras tres décadas del siglo anterior (Álvarez, 2006b; Universidad de Puerto Rico, 1915; 1917; University of Puerto Rico, 1922; 1923; 1924).

La inclinación por el funcionalismo no es de sorprender si consideramos que durante las primeras décadas del siglo XX la Universidad de Columbia en Nueva York, una de las cunas del sistema funcionalista estadounidense, se convirtió en el lugar preferido de los profesores y profesoras del Departamento de Psicología para proseguir sus estudios graduados. De los once profesores que trabajaron en este departamento, nueve realizaron estudios graduados en la Universidad de Columbia.Los egresados de Columbia constituyeron una de las arterias a través de la cual llegó este tipo de psicología a la Isla. Así, una breve acotación para mirar hacia dicha institución puede ser provechosa en la reconstrucción historiográfica del momento.

A finales del siglo XIX y principios del XX, Edward Thorndike, James Mc Catell, John Dewey y Robert Sessions Woodworth coincidieron en Columbia (Boring, 1950/1978). Entre 1917 al 1942, bajo Woodworth en Columbia, se abordaron los tópicos propios de la época: estadísticas, curva de distribución, diferencias individuales, medición de la inteligencia y de otras habilidades humanas, métodos experimentales y fisiología. Se incluyó como parte de las materias las aplicaciones de la psicología a la biología, la industria, el comercio y la educación. Durante este lapso que coincide con los años de formación del profesorado puertorriqueño en la institución, se expuso al estudiantado a diversas líneas de pensamiento y a una menor adhesión hacia el asociacionismo y a la psicología de los estímulos y las respuestas que la observada en otras instituciones estadounidenses (Boring, 1950/1978). Tanto Thorndike, Catell, Dewey y Woodworth fueron privilegiados en los escritos no empíricos de los profesores del departamento de psicología de la Universidad de Puerto Rico, los que se aglomeraron en temas pedagógicos y desarrollo humano. 
Es importante puntualizar que el funcionalismo advino a la Isla previo al establecimiento del departamento de psicología. Fuentes secundarias disponibles sostienen que antes de la década del veinte, las descripciones de los cursos de psicología que eran parte de los currículos de Educación y Artes Liberales denotaban una orientación funcionalista y una preferencia por los métodos experimentales (Álvarez, 2006b). Coincidiendo con los intereses propios de la psicología funcionalista se dedicaron en el departamento grandes esfuerzos hacia la cuantificación de los constructos psicológicos, la inteligencia inclusive. Los profesores se concentraron en la construcción de diversas herramientas de medición. A finales de la década del veinte, Fred Walters - el estadounidense que ejerció como director de departamento desde sus inicios hasta 1942 -junto a Malvina L. Monefeld, Alfredo Silva y Mercedes Chiqués, tradujeron y adaptaron la Stanford Revision of the Binet-Simon Tests (Publications, lectures, studies by Fred Walters, 1939). La StanfordBinet se utilizó en Puerto Rico hasta 1951, cuando bajo los auspicios del Departamento de Instrucción Pública se tradujo y se adaptó la Escala de Inteligencia Wechsler para niños (Roca, 2000).

Con todo, los antecedentes descritos no constituyen el único marco referencial histórico en donde ubicar la producción académica de los profesores del departamento de psicología. El pasado de la psicología en Puerto Rico hay que aprehenderlo en el escenario político-social en el que ocurrió. Resulta significativa la convergencia entre las predilecciones temáticas departamentales y los intereses de los nuevos Comisionados de Educación aquilatados por Aida Negrón de Montilla en su libro La Americanización de Puerto Rico y el Sistema de Instrucción Pública (1900-1930). Según Negrón de Montilla (1977) durante las primeras décadas del siglo XX los titulares de educación se concentraron en la americanización, la ampliación del sistema escolar y la enseñanza del inglés.

De esta forma, se darán en la isla varios procesos calados por la ideología de los recién arribados quienes se veían como portadores del progreso (Negrón de Montilla, 1977; Scarano, 2002). Las transformaciones subvencionadas por el Estado en la educación, la economía, la salud y las obras públicas eran desde la óptica estadounidense mecanismos para el progreso en la nueva posesión insular cuyos habitantes percibían como inferiores cognitivamente e incapaces de autogobernarse (Scarano, 2002). La aprobación de la Ley Foraker en 1900 por el Congreso de los Estados Unidos como base legal para un gobierno civil colonial, la consolidación del capitalismo agrario como sistema económico y los intentos de americanización son puntales importantes para aprehender las transformaciones en la Isla durante las primeras décadas del siglo XX. Con la Ley Foraker, la Isla pasó a ser un territorio no incorporado de los Estados Unidos. En 1917 el Congreso Estadounidense 
sustituyó la Ley Foraker por la Ley Jones. Bajo la Ley Foraker y la Ley Jones el presidente de los Estados Unidos designaba muchos de los funcionarios locales, como el gobernador y el Comisionado de Educación. Tanto el Acta Orgánica Foraker como la Jones, que otorgó la ciudadanía estadounidense a los puertorriqueños, sentaron las bases jurídicas del sistema de educación de Puerto Rico (Negrón de Montilla, 1977).

Posterior al 1898 el sistema de enseñanza público de la Isla, la Universidad de Puerto Rico inclusive, se centralizó bajo el mando del Comisionado de Educación. Este funcionario tenía como encomienda reemplazar el sistema antecedente por uno que asegurara la americanización. En sus manos estaban las decisiones curriculares, programáticas y nombramientos. Hasta 1923, la ley asignó al Comisionado de Educación la cancillería de la universidad. A partir de esta fecha un nuevo estatuto ordenó la designación de un canciller en propiedad para ésta. El Comisionado de Educación presidiría la Junta de Síndicos, organismo rector de la universidad, posición que también llenaba previo a 1923 (González, 1982; Scarano, 2000).

En 1925 con el objetivo de evaluar el sistema educativo del país, la cancillería de la Universidad contrató los servicios de la unidad de investigación del Teachers College de la Universidad de Columbia. Entre otras cosas, el equipo que llegó a la isla "estimó las habilidades mentales de los nuevos ciudadanos americanos" (Roca, 2000, p.59). Se utilizó la Pinter Non Language Ability Test,- primera prueba de inteligencia administrada en la Isla, - y la prueba para medir aprovechamiento académico Stanford Achievement Test. Ambas ya habían sido traducidas por un grupo de profesores del departamento de psicología y el sistema de instrucción pública. Los resultados, que se publicaron bajo el título A survey of the public educational system of Porto Rico, señalaban la conveniencia de establecer un centro de investigación para preparar exámenes que evaluaran las transformaciones educativas que se iban dando en el país. Asimismo, la comisión de Columbia concluyó que las habilidades intelectuales de los puertorriqueños eran similares a las habilidades de las masas urbanas en los Estados Unidos continentales. Y que no tenían reservas en afirmar que los puertorriqueños tenían la capacidad de adquirir educación en un sistema escolar moderno (Columbia University, 1926; Roca, 2000; Rodríguez, 1991).

A partir de 1925 el departamento de psicología amparado en la legitimidad de una psicología científica, objetiva y en la eficacia de sus aplicaciones prácticas, colaboró con la tarea. Sus test contribuyeron a tomar el pulso a las nuevas estrategias educativas implementadas por el gobierno estadounidense. Como señala el decano José Osuna en el prólogo que escribiera para el libro Detalles del Test Hispanoamericano de Habilidad y Aprovechamiento publicado en 1935 por Fred Walters y Mercedes Chiqués Walsh: 
La aplicación de la psicología a la enseñanza constituye uno de los progresos más notables de la pedagogía moderna. Una de las fases más importantes de ese movimiento, tanto en Europa como en los Estados Unidos ha consistido en la preparación de exámenes (test) para medir la inteligencia y el aprovechamiento de los alumnos...Nuestro departamento de psicología se dio inmediata cuenta de esta necesidad...[Sus] exámenes han contribuido eficazmente a la clasificación y orientación de los estudiantes... es decir abordar el problema educativo de una manera más objetiva y científica (p.9).

En resumen, el departamento de psicología se convirtió en un centro para la construcción, distribución y orientación sobre test para las escuelas públicas y privadas del país. Redactaron el Spanish American Test of English y varias formas de la Prueba de Habilidad General para estudiantes universitarios. Entre 1926 al 1934 construyeron seis formas diferentes del Test Hispanoamericano de Habilidad y Aprovechamiento para estudiantes de escuela superior. Para estas tres pruebas estimaron normas y otras características psicométricas (Osuna, s.f.). De igual forma, como parte del programa de medición para la escuela elemental a cargo de Mercedes Chiqués Walsh, hicieron varias versiones del Test Hispanoamericano para alumnos de tercero al octavo grado. A principios de la década del treinta, bajo el señalado programa, se comparó el estatus académico de los niños de la escuela elemental de la Universidad de Puerto Rico con los niños de otras escuelas elementales urbanas. Para ello usaron el Test Hispanoamericano, además del Stanford Achievement Test, el English Reading Comprehension y el Porto Rico Adaptation (Escuela Elemental de la Universidad de Puerto Rico, 1932).

Las publicaciones de carácter empírico del profesorado, divulgadas en su mayoría en Summer School Review se concentraron en los resultados de los aludidos trabajos psicométricos. Algunas de las publicaciones fueron: Sources of error in school marks (1926), A statistical study of certain aspects of the time factor in intelligence (1927), Psychological Testing in Puerto Rico (1927), A brief study of the Predictive Value of the University Ability Test (1929), A translation and adaptation for Puerto Rico of Binet-Simon Test (1930), The utility of measurement (1937) y Detalles del Test Hispanoamericano de Habilidad y Aprovechamiento (1935). Walters y Chiqués escribirían que esta última:

...medía aprovechamiento en las distintas asignaturas, y al mismo tiempo llenaba la necesidad que había de un instrumento de medición de habilidad general (inteligencia). La nota obtenida en el test completo podía interpretarse no 
solo como una medida de aprovechamiento del niño en su trabajo escolar, sino también como una medida de su habilidad para hacer trabajo escolar, aunque no lo estuviera haciendo y en ambos usos podía tenerse confianza (1935, p.11).

Así, a finales de la década del treinta, los informes rendidos por Fred Walters a la administración universitaria subrayaban las iniciativas del departamento para llevar a cabo un estudio en las escuelas que sirviera de base para determinar el nivel de inteligencia de los puertorriqueños (Department of Psychology, 1937).

\section{Consideraciones finales}

La historia de la psicología en Puerto Rico en el periodo que nos ocupa es un tema que se ha trabajado poco. Apenas par de escritos se han concentrado en este lapso. En síntesis, la evidencia documental que hemos recopilado hasta ahora nos permite discurrir algunas conjeturas sobre el momento histórico. En primer lugar, los intereses estadounidenses,-la ampliación del sistema escolar, la americanización y la enseñanza del inglés,- supeditaron en alguna medida las prioridades del primer departamento de psicología. Las pruebas desarrolladas por esta dependencia midieron los giros estudiantiles ante las nuevas estrategias educativas. Adicional a esta línea de investigación en el área de psicometría educativa, la articulación con la Universidad de Columbia, -que no fue exclusiva del departamento de psicología,- y la preferencia por el sistema funcionalista estadounidense caracterizaron estos años. Como cualquier otra materia la evolución de la disciplina estuvo subordinada a factores internos y externos a la ciencia. Elementos socio-políticos marcaron la trayectoria de la disciplina académica en la Isla. La psicología sirvió como ciencia práctica al servicio de los intereses gubernamentales para transformar la educación.

Llama la atención algunos puntos de convergencia entre la psicología en Puerto Rico y el resto de Latinoamérica. Como en otros países de la región, la historia de la psicología en Puerto Rico está fuertemente entrelazada a la pedagogía. Muchos de los pioneros puertorriqueños, al igual que algunos de los latinoamericanos, eran pedagogos atraídos por cuestiones psicológicas (Ardila, 1969, 1986; De la Torre, 1989; Roca, 2006). En Puerto Rico, como en Cuba y la República Dominicana, la ampliación del sistema público de educación favoreció el desarrollo de la psicología (Álvarez, 2006a). I gualmente, durante estos años no solo la psicología en Puerto Rico recibió el impacto estadounidense. Este fenómeno de importación ha sido documentado en trabajos sobre la psicología en el Caribe (Álvarez, 2006a) y 
Latinoamérica (De la Torre, 1989). De acuerdo a De la Torre (1989), la psicología de la adaptación estadounidense, con su filosofía pragmática y su énfasis en la administración de pruebas empezó a ocupar un espacio a partir de la I Guerra Mundial en el ámbito latinoamericano. Entre 1914 hasta 1945 el fortalecimiento de los nexos neocoloniales con Estados Unidos viabilizó la gradual introducción de las vertientes estadounidenses y europeas americanizadas en la región. Por supuesto, las formas e intensidad de la importación de la psicología estadounidense en el periodo que nos ocupa variaron de un país a otro.

Cabe señalar, que las fuentes primarias compiladas no reflejan una fuerte influencia latinoamericana en la psicología en Puerto Rico en las primeras décadas del siglo XX. Más aún, análisis realizados en el país para la década del sesenta todavía hallan una marcada preferencia por la psicología y autores estadounidenses y exiguas referencias a autores latinoamericanos (González, 2007).

Tal y como señalamos al principio lo expuesto son solo las primeras observaciones de una investigación que aspira a concretar y contextualizar la disciplina que cultivaron aquellos que trabajaron en el primer departamento de psicología en la Universidad de Puerto Rico. Nos quedan aun diversos aspectos por ser examinados y ampliados.

\section{Referencias}

ÁlVAREZ, A. La construcción de la historia de la psicología en el Caribe isleño. Fundación Cátedra I beroamericana, 2006a. Disponible en <https://mail.google.com/a/upr.edu/?ui=2\&ik=bcce6ae430\&view>. Accedido el 30 de agosto de 2013.

ÁlVAREZ, A. La enseñanza de la psicología en la Universidad de Puerto Rico, Recinto Río Piedras, 1903-1950. Revista Puertorriqueña de la Psicología, San Juan, v.17, p.93-113, 2006b.

ARDILA, R. Desarrollo de la psicología latinoamericana. Revista Latinoamericana de Psicología, Bogotá, v.1, n.1, p. 63-71,1969. ARDILA, R. La psicología en América Latina. Pasado, presente y futuro. México, D. F.: Siglo XXI, 1986.

BATISTA, A. Preferencias ocupacionales de los estudiantes del Colegio de Pedagogía, Universidad de Puerto Rico. Ponencia presentada en la convención de psicólogos de Puerto Rico, Río Piedras, 1967.

BORING, E. Historia de la psicología experimental. México, D.F.: Editorial Trillas, [1950]1978. 
Columbia University. A survey of the public educational system of Porto Rico. New York: J.J. Little and Ives Company, 1926.

DE LA TORRE, C. Psicología latinoamericana: Entre la independencia y la identidad. San Juan: Publicaciones puertorriqueñas, 1989.

Department of Psychology. Report for the academic year 19361937. (Caja P-31), Archivo Central, Universidad de Puerto Rico, Río Piedras, 1937.

Department of Psychology. Report for the academic year 1939-1940. (Caja P-31), Archivo Central, Universidad de Puerto Rico, Río Piedras, 1940.

Escuela Elemental de la Universidad de Puerto Rico. I nforme Anual para 1931-32. (Caja P-20), Archivo Central, Universidad de Puerto Rico, Río Piedras, 1932.

Escuela Normal Insular. Catálogo y prospecto para el año escolar. Río Piedras, Departamento de Educación, [1902]1903.

GONZÁLEZ, N. Historia de la Universidad de Puerto Rico 19031930.1982. 301p. Tesis (Maestría en Historia)- Universidad de Puerto Rico, Río Piedras, 1982.

GONZÁLEZ, S. Dos décadas de ponencias en la Asociación de Psicología de Puerto Rico: Una mirada a los sesenta y ochenta. Revista Puertorriqueña de Psicología, San Juan, v. 18, p. 1037,2007.

Instituto Civil de Segunda Enseñanza de Puerto Rico. Programa de psicología, lógica y filosofía moral. San Juan: Imprenta del Boletín, 1874.

MASSÓ, G. El desarrollo de la Universidad de Puerto Rico. Compendio de una historia de treinta años. EI Mundo, San Juan, p.7, 14 feb. 1932.

NEGRÓN DE MONTILLA, A. La americanización de Puerto Rico y el sistema de instrucción pública 1900-1930. Barcelona: Editorial Universitaria, 1977.

OSUNA, J. Prólogo. En: F. Walters \& M. Chiqués. Detalles del test hispanoamericano de habilidad y aprovechamiento. Río Piedras: Universidad de Puerto Rico, 1935, p.9-10.

OSUNA, J. Prólogo. Correspondencia General-Oficina de Rector 1935-1936, Caja 60, Archivo Central, Universidad de Puerto Rico, Río Piedras, s.f.

Publications, lectures, studies by Fred Walters (1939). Expediente de Fred Walters, Caja 7), Archivo Central, Universidad de Puerto Rico, Río Piedras, 1939.

ROCA, I. La medición psicológica en Puerto Rico. En: L. Herrans, Psicología y medición. México, D. F.: Mc Graw-Hill Interamericana 2000, p.59-64. 
ROCA, I. Algunos precursores de la psicología en Puerto Rico: Reseñas biográficas. Revista Puertorriqueña de la Psicología, San Juan, v. 17, p. 61-88, 2006.

RODRÍGUEZ, C. Colonial politics and education: The panamericanization of the University of Puerto Rico 1923-1929. Historia y Sociedad, Río Piedras, v. IV, p. 138-164, 1991.

SCARANO, F. Puerto Rico: Cinco siglos de historia. México, D. F.: Mc Graw Hill, 2002.

Universidad de Puerto Rico. Catálogo anual de la Universidad de Puerto Rico. Prospecto para el año 1917-1918. Río Piedras: Author, 1917.

University of Puerto Rico. Annual catalog of the University of Puerto Rico. Announcement for the year 1915-1916. Río Piedras: Author, 1915.

University of Puerto Rico. Summer school announcement. Río Piedras, Department of Education, 1922.

University of Puerto Rico. Summer school announcement. Río Piedras, Department of Education, 1923.

University of Puerto Rico. Summer school announcement. Río Piedras, Department of Education, 1924.

Walters F. y Chiqués, W. Detalles del Test Hispanoamericano de Habilidad y Aprovechamiento. Río Piedras: Universidad de Puerto Rico, 1935.

\section{Endereço para correspondência \\ Sofía González-Rivera \\ 230 Calle Arterial Hostos \\ Condominio Atrium Plaza \\ Apartamento 901, San Juan, Puerto Rico 00918 \\ Endereço eletrônico: sgonzalez266@hotmail.com}

Recebido em: 02/01/2013

Reformulado em: 02/09/2013

Aceito para publicação em: 04/09/2013

Acompanhamento do processo editorial: Ana Maria Jacó-Vilela

\footnotetext{
Notas

* Profesora adjunta de la Universidad del Sagrado Corazón, San Juan, Puerto Rico. Posee un doctorado en psicología de la Universidad de Puerto Rico.

1 Versión revisada de la ponencia presentada en el Simposio La psicología en América Latina en la primera mitad del siglo XX durante el IV Congreso Regional de la Sociedad Interamericana de Psicología en Santa Cruz, Bolivia, el 22 de junio de 2012.

2 El listado de los textos se obtuvo de los catálogos y anuncios de la oferta académica. En estos documentos se especifican los títulos y la descripción de los cursos que ofrecería la Universidad. En algunas ocasiones se precisaba el títuloautor del libro que el estudiante debía adquirir para la clase. La Oficina del Registrador y el Archivo General Histórico de la Universidad de Puerto Rico no conservan copias de todos los catálogos y anuncios de la oferta académica del periodo bajo estudio.
} 